\section{Evolution of cooperation without reciprocity}

\author{
Rick L. Riolo ${ }^{\star}$, Michael D. Cohen $\uparrow$ \& Robert Axelrod $\ddagger$
}

${ }^{*}$ Center for the Study of Complex Systems; and $†$ School of Information; and $\ddagger$ Gerald R. Ford School of Public Policy, University of Michigan, Ann Arbor, Michigan 48109, USA

A long-standing problem in biological and social sciences is to understand the conditions required for the emergence and maintenance of cooperation in evolving populations. For many situations, kin selection ${ }^{1}$ is an adequate explanation, although kinrecognition may still be a problem. Explanations of cooperation between non-kin include continuing interactions that provide a shadow of the future (that is, the expectation of an ongoing relationship) that can sustain reciprocity ${ }^{2-4}$, possibly supported by mechanisms to bias interactions such as embedding the agents in a two-dimensional space ${ }^{4-6}$ or other context-preserving networks ${ }^{7}$. Another explanation, indirect reciprocity ${ }^{8}$, applies when benevolence to one agent increases the chance of receiving help from others. Here we use computer simulations to show that cooperation can arise when agents donate to others who are sufficiently similar to themselves in some arbitrary characteristic. Such a characteristic, or 'tag', can be a marking, display, or other observable trait. Tag-based donation can lead to the emergence of cooperation among agents who have only rudimentary ability to detect environmental signals and, unlike models of direct ${ }^{3,4}$ or indirect reciprocity ${ }^{9,10}$, no memory of past encounters is required.

Tag-based mechanisms may be useful even if an agent is unable to observe or remember others' actions. To show how tag-based strategies of helping can lead to the emergence of cooperation, we use the donor/recipient setting of ref. 10. In this setting agents are paired at random, and one agent has an opportunity to make a costly donation to the other. Unlike models of tag-based cooperation using an iterated 'prisoner's dilemma' setting ${ }^{11-14}$, this setting has no continuing interaction between pairs of agents. In Nowak and Sigmund's model of 'image scoring' ${ }^{10}$, an agent's decision to donate (cooperate) is based on whether the potential recipient is known to be sufficiently generous to others. In our model, an agent's decision to donate depends only on arbitrary 'tags' associated with the agents. In particular, when an agent meets another agent, it decides to help if and only if both tags are sufficiently similar. Because agents interact with only a few randomly selected others from a moderately sized population, there is little chance that a given pair will meet again. Whether or not an agent donates does not affect the likelihood of receiving help from others. Thus there is no reciprocity, either direct or indirect.

In Holland's original formulation ${ }^{12}$, arbitrary, evolving tags could facilitate selective interactions, and thereby be helpful for aggregation and boundary formation. While a tag-matching mechanism can be arbitrarily complex, we use the simplest mechanism. Each agent has two traits, a tag $\tau \in[0,1]$, and a tolerance threshold $T \geq 0$. Initially, tags and tolerance levels are assigned to agents at random, uniformly sampled from $[0,1]$. (In other experiments, we started with high, $T=0.5$, and low, $T=0.005$, tolerances. Except for short initial transients, the results were not substantially different from those reported here.) In each generation, each agent acts as a potential donor with $P$ others chosen at random, with replacement. Thus for $P=3$, each agent has three opportunities to donate, and on average is chosen three times as a potential recipient.

An agent A donates to a potential recipient B only if B's tag is sufficiently similar to A's tag. In particular, A donates only when B's tag is within A's tolerance threshold, $T_{\mathrm{A}}$, namely when $\left|\tau_{\mathrm{A}}-\tau_{\mathrm{B}}\right| \leq T_{\mathrm{A}}$. Thus an agent with a high $T$ will donate to agents with a wide range of tags, while an agent with a very small $T$ will donate only to those whose tags are nearly the same as its own. If A does donate to $\mathrm{B}, \mathrm{A}$ pays a cost, $c$, and $\mathrm{B}$ receives a benefit, $b$. We note that even equality of tags does not make two agents more likely to interact, but if they do interact then one will donate to the other.

After all agents have participated in all pairings in a generation, agents are reproduced on the basis of their score relative to others. The least fit, median fit, and most fit agents have respectively 0,1 and 2 as the expected number of their offspring. This is accomplished by comparing each agent with another randomly chosen agent, and giving an offspring to the one with the higher score. (Another interpretation of this adaptive process is learning in a fixed population. In the learning interpretation, each agent compares itself to another agent, and adopts the other's tag and tolerance if the other's score is higher than its own.) Each offspring is subject to potential mutation which may change the offspring's tag, tolerance or both. With probability 0.1 , the offspring receives a new tag with a value drawn at random in $[0,1]$. Also with probability 0.1 , the tolerance is mutated by adding mean 0 , standard deviation 0.01 gaussian noise to the old tolerance. If the new $T<0$, it is set to 0 . One run of the model consists of 100 agents and 30,000 generations. Each experimental condition is replicated 30 times.

We find that using the tag-based mechanism and adaptive processes described above, a population of agents is able rapidly to establish a substantial degree of cooperation. For example, with $P=3$ pairings per agent per generation, and with cost $c=0.1$ and benefit $b=1.0$, the average donation rate was $73.6 \%$.

Figure 1 shows the dynamics of the donation rate over the first 500 generations of a typical run using these parameters. The average payoff for the population at any time is proportional to the donation rate because each donation results in one agent gaining $b=1.0$ and another agent losing $c=0.1$, for a net gain to the population of 0.9 . The population starts with tags and tolerances uniformly distributed, so the initial average tolerance is about 0.5 , and the initial average donation rate is about $67 \%$. Within a few generations, however, the agents with low tolerances begin to take over the population as they receive benefits from more tolerant agents, but they bear less of the cost because they donate to fewer others. By generation 70 in the run shown, the average tolerance is down to 0.020 , and the donation rate is down to $43 \%$. By chance there are some small groups of agents with similar tags and relatively low tolerances. As these agents prosper and reproduce, their off-

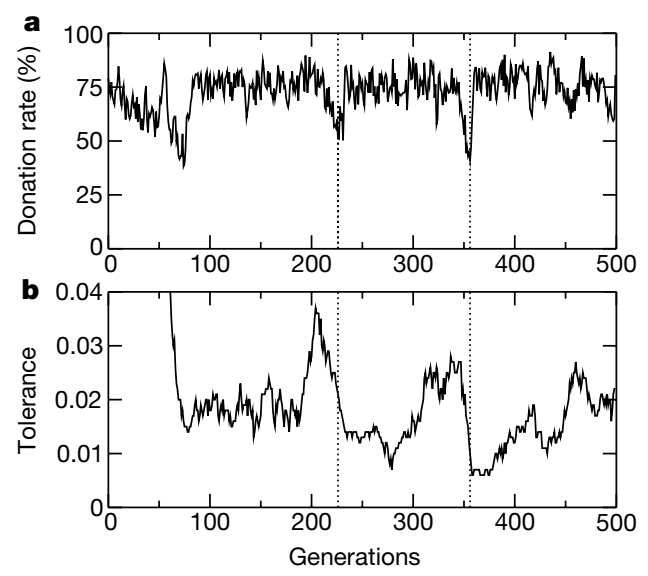

Figure 1 Population dynamics for the first 500 generations of a typical run. a, The donation rate. $\mathbf{b}$, The average tolerance. Occasionally a mutant arises with a tag similar to most of the others, but with an unusually low tolerance. This mutant scores well by receiving donations from many, but donating to few. Its offspring quickly become numerous enough to lower the average donation rate and tolerance of the whole population. Soon their tag becomes the most common, resulting in a transition to a new dominant tag cluster. This happened at generation 226 and 356 . We note that after these transitions, the average donation rate returned to its previous high level. 
spring begin to spread through the population. Soon about $75-80 \%$ of the agents have tags that are so similar that they are within each other's tolerance range. The agents in the resulting 'dominant tag cluster' have an advantage as there are more of them to help each other. The formation of a dominant tag cluster leads to high donation rates even when averaged over the whole population. This establishes the evolution of cooperation without reciprocity.

The dynamics do not end with the establishment of a dominant cluster of similar agents who help each other. For the agents in the dominant cluster, there is only a slight selection pressure to lower their tolerance if there are no agents 'nearby', that is, with a similar tag. The average tolerance can therefore drift upwards owing to mutation occurring near the floor (base value) imposed by $T \geq 0$. Once this happens, the members of the dominant cluster are vulnerable to a relatively intolerant mutant.

The vulnerability of the dominant cluster is realized when a mutant's tag happens to be within the range of tolerance of the typical member of the dominant cluster, but the mutant's own tolerance range is small enough to prevent its donation to members of the dominant cluster. This fortunate but relatively intolerant mutant will then tend to score very well by receiving help from most of those who pair with it, while rarely giving help to others. The result is that the fortunate mutant has many offspring over the next few generations, and soon establishes a new cluster of agents with similar tags and similar (low) tolerances. As the members of this new dominant cluster do not contribute to the old cluster, the average donation rate in the population falls markedly (see Fig. 1). The members of the new cluster donate to each other when they happen to interact because, except for any further mutation of tags, they all inherit the same tag. As the new cluster grows to about 75$80 \%$ of the population, the old cluster dies out and the average donation rate rebounds. The average donation rate recovers quite quickly. This makes possible the overall donation rate of $73.6 \%$ over the entire set of 30 runs of 30,000 generations each.

We can measure the relatedness of a dominant cluster by the proportion that has its modal tag. Excluding the transient period of the first 100 generations, the relatedness of a cluster when it first becomes dominant averages $79 \%$. Ten periods later the dominant cluster's relatedness increases to $97 \%$ as the members who give to the modal type without receiving donations from them are eliminated. Thus, by establishing dominant tag clusters, common descent has a strong influence on the maintenance of cooperation.

The new dominant cluster tends to have relatively low tolerance owing to inheritance from its founder. Over time, the average tolerance of its members tends to drift upwards. In fact, the average tolerance of a dominant cluster is much higher at its end (0.027) than its beginning (0.010). As the tolerance of a new dominant cluster increases, it becomes vulnerable to yet another relatively intolerant mutant with a similar tag. The cycle continues indefinitely. This cycle corresponds to the one found in many models of the iterated 'prisoner's dilemma' among conditional cooperators, unconditional cooperators, and defectors ${ }^{7,13-17}$. In our model, the

\begin{tabular}{lcc}
\hline Table 1 Effect of pairings on donation rate & \\
\hline Pairings & Donation rate (\%) & Average tolerance \\
\hdashline 1 & 2.1 & 0.009 \\
2 & 4.3 & 0.007 \\
3 & 73.6 & 0.019 \\
4 & 76.8 & 0.021 \\
6 & 78.7 & 0.024 \\
8 & 79.2 & 0.025 \\
10 & 79.2 & 0.024
\end{tabular}

We note that increasing the number of pairings of potential donors and recipients per generation increases the donation rate. A potential donor agent $A$ in a pair donates to a potential recipient $B$ only if the distance between the tags of $A$ and $B$ is less than or equal to A's tolerance. Pairings is the number of times per generation each agent has an opportunity to donate to a randomly encountered other. The donation rate is the percentage of such encounters in which the choosing agent cooperates, that is, donates $b=1.0$ at a cost of $c=0.1$ to itself. We note that the donation rate increases markedly between $P=2$ and $P=3$, whereas the average tolerance of the population increases only slightly. cycle of increasing and decreasing tolerance could reflect, for example, a loss of sensory discrimination in a population when there is little selection pressure to retain it, followed by a recovery when a more discriminating individual succeeds.

The success of tag-based donation requires enough pairings per generation to establish dominant clusters. Table 1 shows the effects of varying the number of pairings per agent per generation, $P$, on the donation rate (the amount of cooperation) and on the population's average tolerance $T$. With one or two pairings, the amount of cooperation is less than $5 \%$. With three pairings, the donation rate jumps to $74 \%$, and then rises gradually to $79 \%$ for ten pairings. The sharp transition suggests that it may be possible to approximate these simulation patterns in an analytic model. As agents participate in more pairings, each one has a better chance of being found by an agent that will contribute to it, thus increasing the spread of agents with similar tags in future generations. Higher numbers of pairings also increases the chances that similar agents will continue to find agents to donate to, and also to receive donations, ensuring the formation of a dominant cluster with similar tags.

We note in Table 1 that when there are more than two pairings, the average level of tolerance increases, but only modestly. Thus there continues to be a pressure towards donating only to those with quite similar tags. This pressure is a result of the advantage a relatively intolerant agent has in a group of more tolerant donors with more or less similar tags. The intolerant agent gains fitness because the tolerant agents donate to it, while it bears little cost because the smaller range of tag values to which it will donate means that it will tend to donate to fewer others.

In all these simulations the typical behaviour of the system is attained within a few hundred generations, and then persists stochastically over the entire 30,000 generation period history. That full history is the basis of our reported averages.

The cost/benefit ratio, $c / b$, also affects the rate of donation. Table 2 shows how the donation rate and average tolerance are affected by varying $c / b$ when $P$ is held constant at $P=3$, and all other parameters are unchanged. As expected, the donation rate falls when the relative cost of making donations rises. For costs less than 0.3 , the rate of donation is at a high level. Beyond a cost of 0.4 , the donation rate rapidly collapses.

In our model, each agent compares itself to another agent, and adopts the other's tag and tolerance if the other's score is higher than its own. Suppose instead that the agent adopts the better agent's tag and tolerance with probability proportional to how much better the other agent is. With this method, even one pairing is sufficient to achieve a donation rate of $49 \%$, compared to only $2 \%$ shown in Table 1 . The donation rate and tolerance still decrease with cost, but are less sensitive than before to increases in the number of pairings.

Our model of donation based on similarity of tags extends the insight of Nowak and Sigmund ${ }^{10}$ by reducing the requirements for the participating organisms: a potential donor incurs a certain cost in order to help another individual if and only if their tags are within the donor's range of tolerance. Tags are initially chosen at random, as are tolerances, but both are heritable and subject to mutation. Cooperation based on tag similarity does not require that the agents

\begin{tabular}{|c|c|c|}
\hline Cost & Donation rate (\%) & Average tolerance \\
\hline 0.05 & 73.7 & 0.019 \\
\hline 0.1 & 73.6 & 0.019 \\
\hline 0.2 & 73.6 & 0.018 \\
\hline 0.3 & 73.5 & 0.018 \\
\hline 0.4 & 60.1 & 0.011 \\
\hline 0.5 & 24.7 & 0.007 \\
\hline 0.6 & 2.2 & 0.005 \\
\hline
\end{tabular}

We note that increasing the cost of donating relative to the benefits conveyed decreases the donation rate. Here, the number of pairings per agent per generation is held constant at $P=3$. When agent $A$ donates to agent $B$, the recipient gets $b=1$; the cost/benefit ratio $c / b$ is altered by adjusting the cost, $C$. The donation rate is the percentage of pairings in which an agent cooperates by making a donation. Average tolerance is calculated over the entire population. 
are able to recognize each other from past interactions. Nor does it require that one agent can observe and recall how the other agents behaved with third parties. Therefore cooperation on the basis of similarity could be widely applicable in situations where repeated interactions are rare, and reputations are not established. Indeed, the basis for similarity can be completely arbitrary, such as for chemical markers or cultural attributes. Cultural artefacts that can serve as tags include accents, practices or artefacts subject to fashion such as wearing hats of particular colours ${ }^{18}$. The basis for similarity also can be 'secret handshakes' or other arbitrary behavioural signals that individuals can detect ${ }^{19}$. As an agent does not have to remember previous interactions with another agent, let alone know anything about that agent's behaviour with others, an agent only needs very limited signal-detection capability. Indeed, kin recognition may use tag-based mechanisms such as the 'green beard ${ }^{1,20-23}$ and 'armpit' effects $^{24-28}$. Using tags may also be interpreted as imposing an abstract topology on the agents in which an agent's 'neighbourhood' is defined by its tag and threshold of similarity tolerance ${ }^{14}$. In summary, our results show that cooperation can become established and be sustained even without memory. Not only do the agents not require continuing interactions, they do not even need to observe the behaviour of others or receive reports from third parties. Strategies of donating to others who have sufficiently similar heritable tags-even though such tags are initially arbitrary-can establish cooperation without reciprocity.

Received 3 August; accepted 17 September 2001

Hamilton, W. D. The genetical evolution of social behaviour, I and II. J. Theor. Biol. 7, 1-52 (1964)

2. Trivers, R. The evolution of reciprocal altruism. Q. Rev. Biol. 46, 35-57 (1971).

3. Axelrod, R. \& Hamilton, W. D. The evolution of cooperation. Science 211, 1390-1396 (1981).

4xelrod, R. The Evolution of Cooperation (Basic Books, New York, 1984).

5. Nowak, M. A. \& May, R. M. Evolutionary games and spatial chaos. Nature 359, 826-829 (1992).

6. Lomborg, B. Nucleus and shield: the evolution of social structure in the iterated prisoner's dilemma. Am. Soc. Rev. 61, 278-307 (1996).

7. Cohen, M. D., Riolo, R. L. \& Axelrod, R. The role of social structure in the maintenance of cooperative regimes. Rationality Soc. 13, 5-32 (2001)

8. Alexander, R. D. The Biology of Moral Systems (Aldine de Gruyter, New York, 1987).

9. Boyd, R. \& Richerson, P. J. The evolution of indirect reciprocity. Social Networks 11, 213-236 (1989)

10. Nowak, M. A. \& Sigmund, K. Evolution of indirect reciprocity by image scoring. Nature 393, 573-577 (1998).

11. Axelrod, R. The Evolution of Cooperation 146-150 (Basic Books, New York, 1984).

12. Holland, J. H. Hidden Order: How Adaptation Builds Complexity (Addison Wesley, Reading, Massachusetts, 1995)

13. Riolo, R. L. in Proc. 7th Int. Conf. Genetic Algorithms (ICGA97) (ed. Bäck, T.) 378-385 (Morgan Kaufmann, San Francisco, 1997).

14. Cohen, M. D., Riolo, R. L. \& Axelrod, R. The emergence of social organization in the prisoner's dilemma: how context preservation and other factors promote cooperation. Working paper 99-01-002 (Santa Fe Institute, New Mexico, 1999).

15. Nowak, M. A. \& Sigmund, K. Oscillations in the evolution of reciprocity. J. Theor. Biol. 137, 21-26 (1989).

16. Lindgren, K. in Artificial Life II (eds Langton, C. G. et al.) 295-312 (Addison-Wesley, Reading, Massachusetts, 1991).

17. Linster, B. Evolutionary stability in the infinitely repeated prisoner's dilemma played by two-state Moore machines. South. Econ. J. 58, 880-903 (1992).

18. Allison, P. D. The cultural evolution of beneficent norms. Social Forces 71, 279-301 (1992).

19. Robson, A. J. Efficiency in evolutionary games: Darwin, Nash and the secret handshake. J. Theor. Biol. 144, 379-396 (1990)

20. Dawkins, R. The Selfish Gene 96 (Oxford Univ. Press, Oxford, 1976).

21. Haig, D. Gestational drive and the green-bearded placenta. Proc. Natl Acad. Sci. USA 93, 6547-6551 (1996).

22. Grafen, A. Evolutionary biology—-green beard as death warrant. Nature 394, 521-523 (1998).

23. Keller, L. \& Ross, K. G. Selfish genes: a green beard in the red fire ant. Nature 394, 573-575 (1998).

24. Dawkins, R. The Extended Phenotype 146-151 (Freeman, San Francisco, 1982).

25. Hauber, M. E., Sherman, P. W. \& Paprika, D. Self-referent phenotype matching in a brood-parasite: the armpit effect in brown-headed cowbirds (Molothrus ater). Anim. Cogn. 3, 113-117 (2000).

26. Hauber, M. E. \& Sherman, P. W. The armpit effect in hamster kin recognition. Trends Ecol. Evol. 15 $349-350(2000)$

27. Mateo, J. M. \& Johnston, R. E. Kin recognition and the 'armpit effect': evidence of self-referent phenotype matching. Proc. R. Soc. Lond. B 267, 695-700 (2000).

28. Isles, A. R., Baum, M. J., Ma, D., Keverne, E. B. \& Allen, N. D. Genetic imprinting-urinary odour preferences in mice. Nature 409, 783-784 (2001).

\section{Acknowledgements}

For financial support we thank the Intel Corporation and the University of Michigan College of Literature, Science and the Arts Enrichment Fund. For computing facilities we thank the University of Michigan Center for the Study of Complex Systems.

Correspondence and requests for materials should be addressed to R.L.R

(e-mail: rlriolo@umich.edu).

\section{Effects of experience and social context on prospective caching strategies by scrub jays}

\section{N. J. Emery ${ }^{\star} \&$ N. S. Clayton $\dagger$}

\section{* Sub-department of Animal Behaviour, University of Cambridge, Cambridge} CB3 8AA, UK

$\dagger$ Department of Experimental Psychology, University of Cambridge, Cambridge CB2 3EB, UK

The authors contributed equally to this work

Social life has costs associated with competition for resources such as food ${ }^{1}$. Food storing may reduce this competition as the food can be collected quickly and hidden elsewhere ${ }^{2-4}$; however, it is a risky strategy because caches can be pilfered by others ${ }^{5-9}$. Scrub jays (Aphelocoma coerulescens) remember 'what', 'where' and 'when' they cached ${ }^{10-13}$. Like other corvids ${ }^{6-9,14}$, they remember where conspecifics have cached, pilfering them when given the opportunity, but may also adjust their own caching strategies to minimize potential pilfering. To test this, jays were allowed to cache either in private (when the other bird's view was obscured) or while a conspecific was watching, and then recover their caches in private. Here we show that jays with prior experience of pilfering another bird's caches subsequently re-cached food in new cache sites during recovery trials, but only when they had been observed caching. Jays without pilfering experience did not, even though they had observed other jays caching. Our results suggest that jays relate information about their previous experience as a pilferer to the possibility of future stealing by another bird, and modify their caching strategy accordingly.

In the wild, food-storing corvids return to caches that they had hidden in the presence of conspecifics, and readily re-cache them in new places when the observers are no longer present (for example ravens $^{8,9}$, European jays ${ }^{15}$, scrub jays; N.S.C., unpublished observations). We proposed that birds re-cache to minimize potential pilfering by observers. We therefore predicted that they would be more likely to re-cache any uneaten food, and specifically in new sites unbeknown to an observer, but only if they had been watched during the caching trial. To test this hypothesis, scrub jays were allowed to cache wax worms in a sand-filled caching tray during two

\begin{tabular}{|c|c|c|c|c|c|}
\hline \multirow[b]{2}{*}{ Behaviour } & \multicolumn{2}{|c|}{ Caching treatment } & \multicolumn{3}{|c|}{ Wilcoxon pairs test } \\
\hline & Observed & In private & $n$ & $Z$ & $P$ \\
\hline \multicolumn{6}{|l|}{ No. cached } \\
\hline Davis & $8.19 \pm 1.55$ & $4.71 \pm 0.81$ & 7 & 2.37 & $<0.05$ \\
\hline Cambridge & $10.48 \pm 3.43$ & $9.10 \pm 3.05$ & 7 & 0.51 & $>0.5$ \\
\hline \multicolumn{6}{|l|}{ No. recovered } \\
\hline Davis & $4.61 \pm 0.93$ & $3.95 \pm 0.84$ & 7 & 0.85 & $>0.1$ \\
\hline Cambridge & $5.38 \pm 1.56$ & $4.19 \pm 1.10$ & 7 & 0.08 & $>0.5$ \\
\hline \multicolumn{6}{|c|}{ Proportion recovered } \\
\hline Davis & $0.71 \pm 0.06$ & $0.57 \pm 0.07$ & 7 & 2.03 & $<0.05$ \\
\hline Cambridge & $0.56 \pm 0.08$ & $0.70 \pm 0.09$ & 7 & 1.69 & $>0.05$ \\
\hline \multicolumn{6}{|c|}{ Recovery accuracy* } \\
\hline Davis & $2.21 \pm 0.46$ & $3.21 \pm 1.01$ & 7 & 1.15 & $>0.1$ \\
\hline Cambridge & $3.07 \pm 0.92$ & $1.52 \pm 0.24$ & 7 & 1.36 & $>0.1$ \\
\hline \multicolumn{6}{|l|}{ No. re-cached } \\
\hline Davis & $2.19 \pm 0.68$ & $0.57 \pm 0.32$ & 7 & 2.20 & $<0.05$ \\
\hline Cambridge & $2.74 \pm 1.01$ & $0.36 \pm 0.19$ & 7 & 2.20 & $<0.05$ \\
\hline \multicolumn{6}{|c|}{ Proportion re-cached } \\
\hline Davis & $0.44 \pm 0.20$ & $0.06 \pm 0.03$ & 7 & 2.20 & $<0.05$ \\
\hline Cambridge & $0.28 \pm 0.07$ & $0.08 \pm 0.04$ & 7 & 2.20 & $<0.05$ \\
\hline
\end{tabular}

Treatments consisted of three trials/caching treatment at Davis, followed by three trials/caching treatment at Cambridge (data are mean \pm s.e.m.). A Wilcoxon matched-pairs test compared the effect of caching treatment for each of the behaviours listed. effect of caching treatment for each
${ }^{*}$ Number of looks to find first cache. 